\title{
Higher cortisol levels may proceed a manic episode and are related to disease severity in patients with bipolar disorder
}

\author{
Monique T. van den Berg ${ }^{\mathrm{a}, *}$, Vincent L. Wester ${ }^{\mathrm{b}}$, Annabel Vreeker ${ }^{\mathrm{c}}$, Manja A. Koenders ${ }^{\mathrm{a}}$, \\ Marco P. Boks ${ }^{\text {, }}$, Elisabeth F.C. van Rossum ${ }^{\mathrm{b}}$, Anne T. Spijker ${ }^{\mathrm{a}}$ \\ ${ }^{a}$ Department of Mood Disorders, Parnassia NAH, Rotterdam, The Netherlands \\ ${ }^{\mathrm{b}}$ Department of Internal Medicine, Division of Endocrinology, Erasmus MC, University Medical Center Rotterdam, Rotterdam, The Netherlands \\ ${ }^{\mathrm{c}}$ Brain Center Rudolf Magnus, Department of Psychiatry, Medical Center Utrecht, Utrecht, The Netherlands
}

\section{A R T I C L E I N F O}

\section{Keywords:}

Hair cortisol concentrations

Bipolar disorder

Manic episodes

Mood

Severity

\begin{abstract}
A B S T R A C T
Background: Regulation of the hypothalamic-pituitary-adrenal (HPA) axis is implicated in the pathogenesis of bipolar disorder (BD). However, the relationship between HPA-activity and disease severity is not fully elucidated. In this pilot study we aimed to explore the temporal relationship between HPA-activity and the risk of a manic episode in BD patients type I, by assessing long-term hair cortisol concentrations (HCC). Second, we explored the relation between HCC and the number of previous episodes.

Methods: Hair samples were collected from 45 BD I patients in euthymic or manic state and compared to 17 controls. From each participant, two hair samples of $3 \mathrm{~cm}$ length were used to measure long-term cortisol, reflecting retrospect time frames of 1-3 months and 4-6 months respectively prior to sampling. Results: HCC in the BD group was slightly higher than in the control group in both hair segments ( $\mathrm{p}=0.049$ and 0.03; after adjustment for age, sex, BMI and hair washing frequency $\mathrm{p}=0.222$ and 0.139 ). A significant peak in hair cortisol was observed prior to a manic episode $(\mathrm{p}=0.036)$. Furthermore, we found a positive correlation between the number of mood episodes HCC $(\mathrm{p}=0.03)$.

Conclusions: Our results indicate that long-term cortisol levels are slightly higher in BD, and in particular elevated in the months prior to a manic relapse. In addition HCC are positively associated with the number of previous mood episodes in the course of BD type I.
\end{abstract}

\section{Introduction}

Bipolar Disorder (BD) is a severe mental illness, characterized by a chronic and unpredictable course. Understanding the pathophysiological processes is crucial to identify new potential therapeutic targets. Current pharmacological and psychotherapeutic treatment results in long term stability in only one third of BD patients, leaving two thirds in urgent need of novel treatment options (Renes et al., 2014).

Endocrine systems seem to play a role in the pathogenesis of BD. The hypothalamus - pituitary - adrenal (HPA) axis, as one of the key systems, shows an altered functioning, characterized by higher cortisol levels (Belvederi Murri et al., 2016; Cervantes et al., 2001; Daban et al., 2005; Deshauer et al., 2003; Jabben et al., 2011; Langan, McDonald 2009; Linkowski et al., 1994; Schmider et al., 1995) a decrease of number of glucocorticoid receptors (Bei et al., 2009; Fries et al., 2014) and a decreased sensitivity in the negative feedback response (Vieta et al., 1999; Watson et al., 2004) in BD patients as compared to healthy individuals. This has been found during episodes (state phenomenon) and in between episodes (trait phenomenon). Results also indicate a role in the staging of the disease, with a more severe dysregulation in those with a higher number of mood episodes (Watson et al., 2004), suggesting chronicity of both endocrine as mood dysregulation.

However, results concerning HPA-axis functioning in BD have been conflicting and are difficult to interpret and compare across studies, which may be due to 1) heterogeneity of patient groups, with differences in disease type, phase and stage, and, 2) methodological issues regarding the assessment of the functioning of the HPA-axis by using time point measures of cortisol in serum or saliva which are under the influence of the circadian rhythm, diurnal pulsatile secretion, and acute psychological or physiological stress. These constantly changing levels complicate the interpretation of serum and saliva cortisol measurements. By using analysis of cortisol in scalp hair, as a method to monitor chronic HPA axis functioning, we can solve these shortcomings of time point measures. This technique measuring hair cortisol concentrations

\footnotetext{
* Corresponding author at: Neuropsychiatry, Parnassia Antesgroep, Rotterdam, Franz Lehartstraat 141, 3069MN Rotterdam, The Netherlands.

E-mail address: monique.vandenberg@parnassiagroep.nl (M.T. van den Berg).
} 
(HCC) has been proven to be a useful marker of long-term systemic cortisol. (Sauvé et al., 2007; van Uum et al., 2008; Manenschijn et al., 2011)

Until now, only few studies have been published reporting HCC in BD. Our group previously observed higher HCC in BD patients with an older age of onset (Manenschijn et al., 2011). Streit et al. (2016) found higher HCC in BD patients compared to controls (CON) and also found a correlation between manic symptoms and HCC.

The aim of this study is to explore long-term cortisol in relationship to relapses to a manic episode. We included an extensively defined cohort of BD type I patients in a euthymic state, either with or without a manic episode prior to hair sampling in order to distinguish between state and trait. In addition, we explored the severity of the disease, by studying the number of mood episodes and long-term cortisol exposure.

\section{Materials and methods}

\subsection{Study design}

This study is part of a large case-control study, the Dutch Bipolar Cohort (DBC), investigating genetic and phenotypic information of patients with bipolar disorder type I, first degree relatives and controls (CON). The study design is described in more detail by Vreeker et al. (2016), [PMID: 30303059]. In brief, patients were recruited via clinicians, the Dutch patients' association for BD, pharmacies and advertisements. Healthy controls were recruited via advertisements and among individuals who previously participated in scientific studies and agreed to be contacted for new research. Inclusion criteria were: 1) age $\geq 18$ years, 2) at least three Dutch-born grandparents, 3) a good understanding of the Dutch language. Participants were considered to be euthymic when they did not meet DSM-IV criteria for a mood episode, according to the Structured Clinical Interview for DSM-IV (SCID-I). Patients with somatic illness that could have influenced the diagnosis of BD were excluded. This study was approved by the institutional review board of UMCU. All participants provided written informed consent, and all study procedures were executed in accordance with GCP guidelines.

\subsection{Participants}

As shown in Fig. 1 we included all BD patients with a manic episode in the 6 months prior to the baseline measurement, which were a total of $41 \mathrm{BD}$ patients. In addition, we selected 50 euthymic BD patients and 50 controls (CON), randomly using SPSS.

The patient group with a manic episode was divided in those with a manic episode until 3 months prior the baseline measurement $(\mathrm{N}=$ 21); and those with a manic episode 4-6 months prior to the baseline measurement $(\mathrm{N}=20)$ (see Fig. 1). From these 20 patients, 9 were excluded (6 hair samples were missing, and 3 samples were not suitable for analysis due to low hair weight). In order to create equal groups we selected 17 hair samples for each group, BD patients and CON, selected by optimal hair length and thickness.

\subsection{Patient characteristics and mental health}

At baseline, all participants were interviewed by a trained interviewer at the UMC Utrecht or one of their academic collaborative institutes to collect sociodemographic data, patient characteristics, somatic and psychiatric disease characteristics.

CON were interviewed using the Mini International Neuropsychiatric Interview (Sheehan et al., 1998). CON with a psychotic or bipolar disorder, or with first-degree relatives with psychosis or $\mathrm{BD}$, were excluded. BD patients were diagnosed using the Structured Clinical Interview for DSM-IV Axis I disorders (SCID-I) by First et al. (2002).

Age of onset was defined as age of first medication for the bipolar disorder as reported in the Questionnaire of Bipolar Illness (QBP-NL ; an adaptation of the Enrolment Questionnaire (Stanley Foundation Bipolar Network). (Leverich et al., 2001; Suppes et al., 2001) The number of episodes was defined using the QBP-NL as well.

All participants additionally filled out a questionnaire concerning natural hair color, hair treatment (dyeing/bleaching/permanent), the use of hair products on the day of sampling, the frequency of hair washing (Stadler et al., 2013; Staufenbiel et al., 2015) and the use of corticosteroids, as these are known to influence hair cortisol.

\subsection{Hair sample collection}

A scalp hair sample of approximately 100-150 hairs was obtained from each participant at baseline. Samples were cut as close to the scalp as possible at the posterior vertex. Each hair sample was taped on a piece of paper with the proximal side marked, and stored on room temperature till analysis (Fig. 2).

\subsection{Hair sample preparation work-up and analysis}

Each hair lock was divided into two segments: the proximal $0-3 \mathrm{~cm}$ measured from the scalp, corresponding to the most recent 3 months before collection, and the $3-6 \mathrm{~cm}$ measured from the scalp, corresponding to the period 4-6 months before hair collection. Hair segments were further processed and analyzed separately as described previously by Noppe et al. (2015).

In brief, from each hair segment approximately $12 \mathrm{mg}$ was weighed. Hair segments were washed in $2 \mathrm{~mL}$ of LC-MS grade isopropanol for 2 $\mathrm{min}$, and left to dry. Extraction of steroids took place in $1.4 \mathrm{~mL}$ of LC-MS grade methanol and $100 \mu \mathrm{L}$ of internal standard, at 25 degrees Celsius while the samples were gently shaken. After extraction, samples were centrifuged at $4500 \mathrm{RPM}$ for $5 \mathrm{~min}$ and $900 \mu \mathrm{L}$ of the supernatant was transferred to a clean glass tube. We then added $750 \mu \mathrm{L}$ of LC-MS grade methanol to the remaining supernatant and hair sample, and after centrifugation we transferred an additional $900 \mu \mathrm{L}$ of clear supernatant, resulting in a $1.8 \mathrm{~mL}$ extract per hair segment. Extracts were purified using solid phase extraction, and hair cortisol was quantified on a Water Xevo TS-Q LC-MS/MS system (Waters, Milford MA).

\subsection{Statistical analysis}

Data were analyzed using SPSS 22.0 for Windows (IBM, Armonk, NY). Differences between group characteristics were tested using Chisquare and Mann Whitney $U$ test because of non-normal distributions.

After logarithmic transformation, hair cortisol levels were normally distributed. HCC are expressed as standardized means and $95 \%$ Confidence Interval. HCC between BD patients and CON were compared using independent sample $t$-tests and ANCOVA, adjusted for the following covariates; age, sex, BMI, hair washing frequency (Wester and van Rossum, 2015). Linear regression analyses were conducted in the BD group for the association between the number of different mood episodes and HCC. A two-sided p value below 0.05 was considered statistically significant.

\section{Results}

In total, $45 \mathrm{BD}$ patients and $17 \mathrm{CON}$ were enrolled in this study (Fig. 1). No differences between groups were found regarding demographics, BMI, corticosteroid use and hair characteristics (Table 1).

\subsection{Bipolar patients versus healthy controls}

Slightly higher HCC were found in BD patients compared to CON (Fig. 3). In the period 1 through 3 months prior sampling, HCC standardized mean in BD was: $5.12 \mathrm{pg} / \mathrm{mg}$ hair (95 \% CI: 3.92-6.70) (standardized $\beta$ 0.265; $p=0.048$ ). After adjustment for sex, age, BMI 


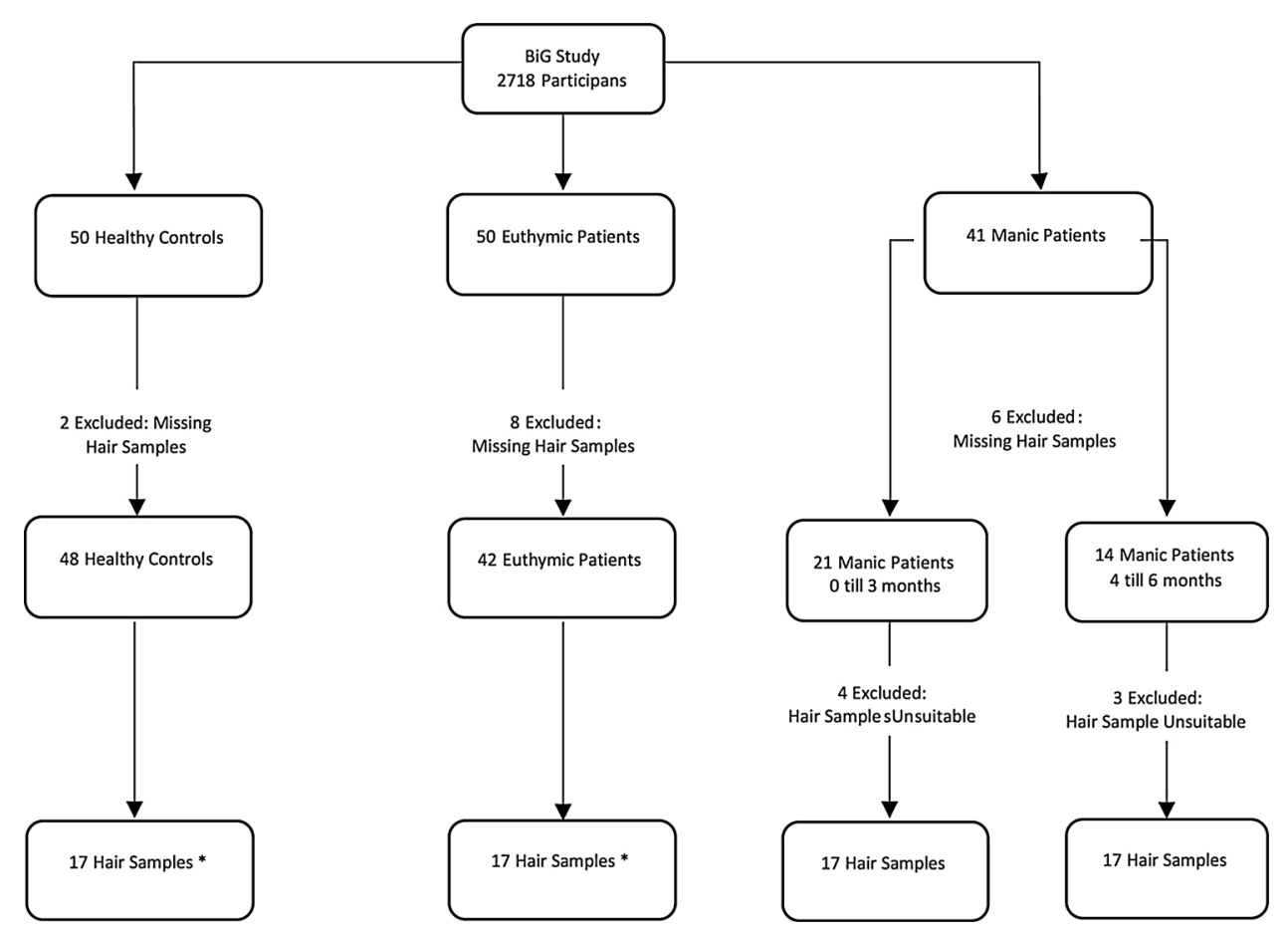

Fig. 1. Flow chart study population.

*Hair weight too low.

\section{Months prior to sampling}

Hair Segment

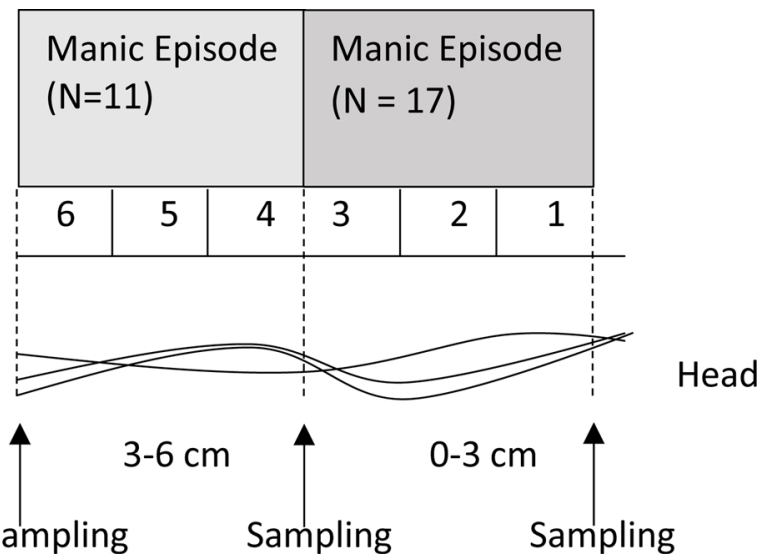

Fig. 2. Hair sample analyses. and hair washing frequency the standardized $\beta$ was $0.164 ; \mathrm{p}=0.185$. In CON the standardized mean was $3.12 \mathrm{pg} / \mathrm{mg}$ hair ( $95 \%$ Confidence Interval (CI):1.96-4.93) (Fig. 3). In the period 4 through 6 months prior to sampling, HCC standardized mean in BD was: $3.97 \mathrm{pg} / \mathrm{mg}$ (95 \% CI: 2.83-5.57)(standardized $\beta$ 0.293; $p=0.031$ ). After adjustment for sex, age, BMI and hair washing frequency $\beta$ is $0.197 ; \mathrm{p}=0.118$. In CON the standardized mean was $2.03 \mathrm{pg} / \mathrm{mg}$ hair (95 \% CI: $1.29-3.20$ ).

Furthermore, we found higher HCC in the hair samples representing 4 through 6 months prior to sampling in those BD patients with a manic episode 1 through 3 months prior to sampling as compared to the hair samples representing 1 through 3 months. This is indicating a preceding peak in the cortisol level followed by a manic episode $(\beta=0.834 ; \mathrm{p}=$ 0.041 after correction $\beta=0.732 ; p=0.118$ ) (Fig. 3). No differences were found between the different disease states.

\subsubsection{Number of episodes}

A positive association was found between the number of mood episodes before study entry and HCC (standardized $\beta=0.462, \mathrm{p}=$ 0.005 ( 0 till 3 months prior sampling) and standardized $\beta=0.382, \mathrm{p}=$
0.032 (4 till 6 months prior sampling)). This association was shown for both manic as well as depressive episodes (Table 2).

\section{Discussion}

In this pilot study we found a relationship between increased Hair Cortisol Concentrations (HCC), indicative of increased HPA activity, and a higher probability of a manic episode in BD patients type I. Second, we show a positive association between HCC and the number of previous episodes, representing the disease chronicity.

In the current study we aimed to unravel whether increased cortisol exposure is a condition preceding a mood episode or rather a consequence of a manic or depressive episode. Our data indicate a potential temporal relationship between long-term cortisol and direct risk of (manic) relapse in disease. We observed higher HCC in the patient group with a recent manic episode (1 through 3 months prior to sampling) based on a higher HCC in the hair sample representing the period 4 through 6 months, which suggest a peak in cortisol occurs prior to a manic episode. However, after correction for the most common 
Table 1

Demographic characteristics of sample.

\begin{tabular}{|c|c|c|c|}
\hline & $\begin{array}{l}\text { Total group } \\
\text { Controls } \\
(\mathrm{N}=17)\end{array}$ & $\begin{array}{l}\text { Total group } \\
\text { Bipolar patients } \\
(\mathrm{N}=45)\end{array}$ & $\mathrm{P}$ - value \\
\hline \multicolumn{4}{|l|}{ Sociodemographics } \\
\hline Male; n (\%) & $3(17.6)$ & $14(31.1)$ & 0.289 \\
\hline Age (year); median (IQR) & $41(25.5-48.0)$ & $46(36.0-53.0)$ & 0.136 \\
\hline \multicolumn{4}{|l|}{ Health Factors } \\
\hline BMI; mean (IQR) & $\begin{array}{l}26.2 \\
(23.5-27.9)\end{array}$ & $\begin{array}{l}26.9 \\
(24.8-31.7)\end{array}$ & 0.136 \\
\hline Use of Corticosteroids ${ }^{\mathrm{a}}(\%)$ & $1(5.9)$ & $0(0)$ & 0.104 \\
\hline \multicolumn{4}{|l|}{ Hair characteristics } \\
\hline \multicolumn{4}{|l|}{ Hair color (\%) } \\
\hline Black & $0(0)$ & $4(8.9)$ & 0.210 \\
\hline Brown & $5(29.4)$ & $19(42.2)$ & 0.364 \\
\hline Blond & $9(52.9)$ & $16(35.6)$ & 0.220 \\
\hline Grey & $3(17.6)$ & $5(11.1)$ & 0.501 \\
\hline Hair treatment (\%) & $5(29.4)$ & $22(48.9)$ & 0.168 \\
\hline Use of Hair products (\%) ${ }^{\mathrm{b}}$ & $9(52.9)$ & $19(42.2)$ & 0.651 \\
\hline \multicolumn{4}{|l|}{ Hair washing; times a week (\%) } \\
\hline $1-2$ times & $3(17.6)$ & $14(31.1)$ & 0.297 \\
\hline $3-4$ times & $8(47.1)$ & $15(33.3)$ & 0.326 \\
\hline$>4$ times & $6(35.3)$ & $15(33.3)$ & 0.887 \\
\hline \multicolumn{4}{|l|}{ Disease characteristics } \\
\hline Age of Onset (years); median & & 28.5 & \\
\hline (IQR) & & $(21.3-37.0)$ & \\
\hline $\begin{array}{l}\text { Number of episodes: total; } \\
\text { median (IQR) }\end{array}$ & & $6.0(5.0-11.0)$ & \\
\hline : manic episodes; median (IQR) & & $4.0(3.0-5.0)$ & \\
\hline
\end{tabular}

Abbreviations: P, P-value < 0.05; IQR, Interquartile Range.

a Fluticasonpropionaat (nasal spray).

b Hairproducts: hairspray, wax, mouse, gel and other.

covariates (age, sex, BMI, hair washing frequency) (Wester and van Rossum, 2015) the higher HCC did not reach significance. Correction for medication didn't influence the outcome and lowers the power of analyses. The higher HCC is in line with the study of Vieta et al. (1999) who compared 42 lithium treated remitted bipolar I patients to 21 healthy controls in adrenocorticotropic hormone (ACTH) response after a corticotropin-releasing hormone (CRH) stimulation and found an enhanced response in the BD group (higher baseline ACTH). When comparing the patients who relapsed to patients who did not, a significantly higher ACTH response to CRH was seen in the period before (hypo)manic symptoms became clinically apparent.

With respect to disease severity (or chronicity), we found a relationship between higher HCC and the severity of BD, quantified by
Table 2

Overview of predictors for Hair Cortisol Concentration (HCC).

\begin{tabular}{|c|c|c|c|c|c|}
\hline & & \multicolumn{2}{|c|}{$\begin{array}{l}\text { HCC in } 0 \text { till } 3 \\
\text { months prior } \\
\text { sampling }\end{array}$} & \multicolumn{2}{|c|}{$\begin{array}{l}\text { HCC in } 4 \text { till } 6 \text { months } \\
\text { prior sampling }\end{array}$} \\
\hline & & $\beta$ & P-value & $\beta$ & P-value \\
\hline \multirow[t]{2}{*}{ Bipolar disorder (yes or no) } & & 0.265 & 0.049 & 0.293 & 0.030 \\
\hline & * & 0.164 & 0.222 & 0.197 & 0.139 \\
\hline \multirow[t]{6}{*}{ Number of mood episodes } & total & 0.532 & 0.002 & 0.496 & 0.010 \\
\hline & $*$ & 0.462 & 0.005 & 0.382 & 0.032 \\
\hline & manic & 0.421 & 0.012 & 0.397 & 0.020 \\
\hline & $*$ & 0.364 & 0.013 & 0.347 & 0.022 \\
\hline & depressive & 0.533 & 0.002 & 0.419 & 0.024 \\
\hline & $*$ & 0.413 & 0.011 & 0.276 & 0.118 \\
\hline
\end{tabular}

Abbreviations: HCC, Hair Cortisol Concentration in picogram per miligram (pg/ $\mathrm{mg}$ ); Number of mood episodes, number of mood episodes in life time definite by Structured Clinical Interview for DSM-IV axis I diagnoses (SCID-I); $\beta$, beta was calculated with logistic regression; P-value $<0.05$.

*Adjusted: for age, sex, BMI and hair washing frequency.

the number of depressive and manic episodes. This is in line with findings by Fries et al. (2014), regarding a higher (post-dexamethasone) cortisol level in the late stage BD patients; in those with a higher number of episodes and a reduced glucocorticoid receptor activity.

Until now, only a few published studies measured long-term cortisol using hair analysis (HCC) in BD patients ${ }^{16}{ }^{17}$. In our own previous work, Manenschijn et al. (2011), we compared 195 healthy controls and 100 BD patients with different subtypes and different disease states. The study showed significantly higher cortisol levels in BD patients with an age of onset older than 30 years, but no relationship in the earlier onset BD patients. In addition, an association with manic episodes could not be evaluated due to a lack of patients with a recent manic episode. In contrast to our study, Manenschijn et al. included both BD type I and II patients with as well recent depressed and hypomanic episodes. In the present cohort only BD type I patients with a reliably diagnosed manic episode were included, increasing homogeneity which helps to understand the underlying switch process.

Another study by Streit et al. (2016) compared 61 BD type I patients (38 inpatients, 23 outpatients) with 159 schizophrenia patients and 82 healthy controls. They found increased HCC in BD patients compared to healthy controls and higher HCC in BD inpatients than outpatients which indicated a relationship with mood episodes.

Also contra dictionary results were found, as Maripuu et al. (2017) found a negative association between HPA-axis activity and age in bipolar patients, especially those with a longer disease duration and without prophylactic medication. Maripuu et al. compared 159 bipolar
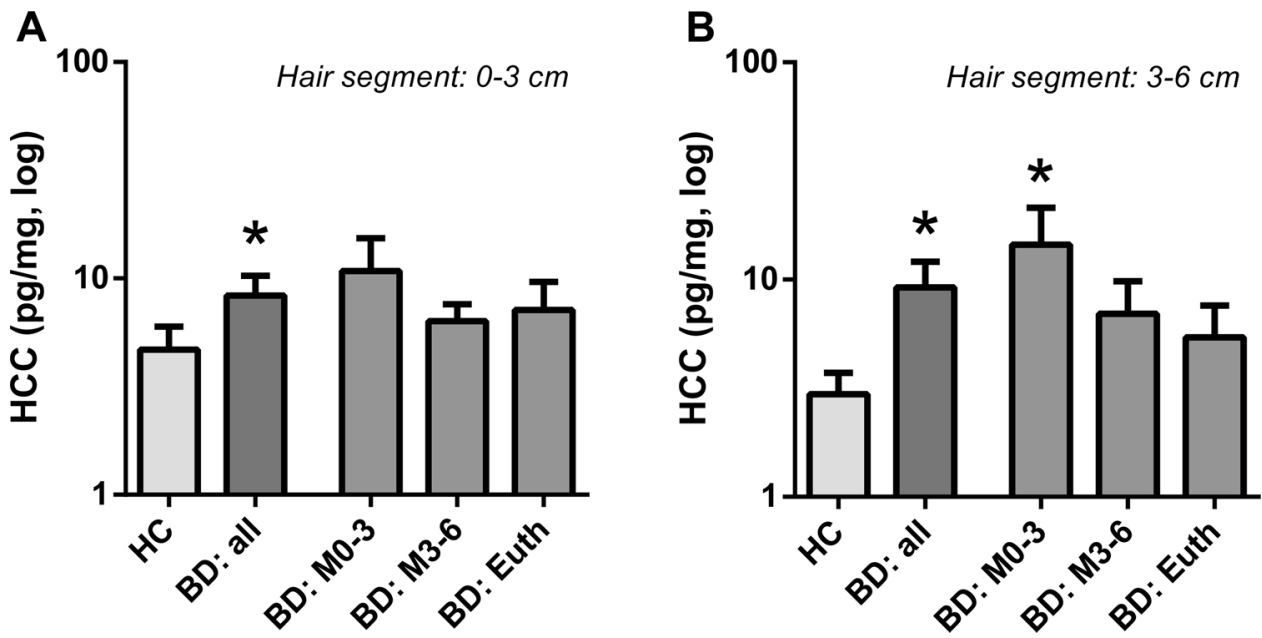

Fig. 3. Results hair cortisol concentration without correction.

HCC in logarithmic scale; Geometric mean \pm SEM; * $\mathrm{p}<0.05$.

CON; BD Bipolar Disorder; BD: M 1-3 Bipolar Disorder with a manic episode in the period 1 through 3 months prior sampling $(\mathrm{N}=17)$; BD: M 4-6 Bipolar Disorder with a manic episode in the period 4 till 6 months prior sampling ( $\mathrm{N}=11)$; BD: Euth: Bipolar Disorder euthymic state. 
out patients and 258 controls.

The observed peak in cortisol prior to a manic episode is a unique finding indicating (hyper-) activation of the HPA-axis preceding a manic episode. The question then arises whether this elevation is causing the subsequent mood episode, or maybe both the increasing cortisol as well as the mood episode are triggered by for example environmental stress. Based on findings in somatic diseases like Cushing's disease, in which the severe hypercortisolism preceeds the mood disorders, it is biologically plausible that also in bipolar disorder a biological dysregulation of the HPA-axis induces the mood episode. Moreover, the relationship between late stage bipolar disorder (as reflected by a higher number of episodes) and higher longterm cortisol levels and number of episodes underlines the interconnectedness of the endocrine process of cortisol regulation and mood stability, with the risk of chronicity of dysregulation of both cortisol and mood dysregulation. This study has several strengths. First, we investigated the relationship between HCC and BD and its mood episodes using a reliable long-term measure of cortisol. HCC provide insight into the chronic potential dysregulation of the HPA axis rather than acute measurements as has been used in earlier research. Hair cortisol analysis seems more suitable to provide this long-term estimate, as they are not influenced by the circadian rhythm, the pulsatile secretion of cortisol and the fluctuation by stress reactions as cortisol in saliva or serum. HCC pave the way to a "timeline approach", showing the interrelationships between cortisol and mood episodes over time. Second, we included a relatively homogeneous and very well defined group of BD type I patients and therefore avoided the potential pathophysiological differences between the subtypes of $\mathrm{BD}$, as we previously found in our studies in BD patients ${ }^{16}$. Third, we used a LC-MS/MS based method, which the current golden standard for measuring steroid hormones in scalp hair such as HCC (Russell et al., 2015).

The first and most important limitation of this study is the small sample size which is directly related to the main question of the study, understanding the role of the HPA-axis in extensively defined and well described cohort of BD type I patients with a manic or euthymic episode in the last six months prior to inclusion. This requires the recruitment of a large cohort of BD patients, as we did, yielding the relatively low, but well phenotyped, numbers of specifically BD type I patients. The second argument to agree with this sample size was the purpose of the study, as it was set up as a pilot study. In this line we accepted the lack of significance in the adjusted analyses.

The second limitation is the lack of repeated measurements for the mood severity. This information will have given us better insight in the changes of mood, as well as the determination of the euthymic state.

Third and last limitation are the (lack of) exclusion criteria for comorbidities and is connected to the first and most important limitation. After excluding the comorbidities from analyses the basic outcome stays the same although with loss of power of the statistical analyses.

To conclude, in the present study we found preliminary evidence for a relation between higher HCC and the risk of a manic relapse as well as a higher number of previous mood episodes in the course of BD type I. However, larger samples are needed to confirm our findings. This might prelude on future studies to cortisol levels in relation to mood episodes in BD including timelines. Because of the potential serious side effects of the manic episode during the course of $\mathrm{BD}$, our finding is promising in providing new options in optimizing treatment of BD by early recognition of vulnerable periods.

\section{Declaration of Competing Interest}

None.

\section{Financial disclosures}

None.

\section{Acknowledgments}

EFCvR is supported by an Erasmus MC research fellowship, a fellowship of the Netherlands Brain Foundation (grant number F2011(1)12), and a Vidi grant of the Netherlands Organization for Scientific Research (grant number 91716453). The Dutch Bipolar Cohort was supported by the National Institute of Mental Health (Grant number: R01MH 090 553).

\section{References}

Bei, E., Salpeas, V., Pappa, D., Anagnostara, C., Alevizos, V., Moutsatsou, P., 2009. Phosphorylation status of glucocorticoid receptor, heat shock protein 70 , cytochroom $\mathrm{C}$ and Bax in lymphocytes of euthymic, depressed and manic bipolar patients. Psychoneuroendocrinology 34 (8), 1162-1175. https://doi.org/10.1016/j.psyneuen. 2009.03.002.

Belvederi Murri, M., Prestia, D., Mondelli, V., Pariante, C., Patti, S., Olivieri, B., 2016. The HPA axis in bipolar disorder: systematic review and meta-analysis.

Psychoneuroendocrinology 63, 327-342. https://doi.org/10.1016/j.psyneuen.2015. 10.014.

Cervantes, P., Gelber, S., Kin, F.N., Nair, V.N., Schwartz, G., 2001. Circadian secretion of cortisol in bipolar disorder. J. Psychiatry Neurosc. 26, 411-416 PubMed PMID: 11762208; PubMed Central PMCID: PMC167199.

Daban, C., Vieta, E., Mackin, P., Young, A.H., 2005. Hypothalamic-pituitary-adrenal axis and bipolar disorder. Psychiatr. Clin. North Am. 28, 469-480. https://doi.org/10. 1016/j.psc.2005.01.005.

Deshauer, D., Duffy, A., Alda, M., Grof, E., Albuquerque, J., Grof, P., 2003. The cortisol awakening response in bipolar illness: a pilot study. Can. J. Psychiatry 48, 462-466.

First, M.B., Spitzer, R.L., Gibbon, M., Williams, J.B.W., 2002. Structured clinical interview for DSM-IV-TR axis I disorders, research version, non-patient edition. (SCID-I/NP) New York. Biometrics Research. New York State Psychiatric Institutehttps://doi.org/ 10.1007/978-0-387-79948-3 2011.

Fries, G.R., Vasconcelos-Moreno, M.P., Gubert, C., dos Santos, B.T., Sartori, J., Eisele, B., et al., 2014. Hypothalamus - Pituitary - Adrenal axis dysfunction and illness progression in bipolar disorder. Int. J. Neuropsychopharmacol. 31, 18. https://doi.org/ 10.1093/ijnp/pyu043.

Jabben, N., Nolen, W.A., Smit, J.H., Vreeburg, S.A., Beekman, A.T.F., Penninx, B.W.J.H., 2011. Co-occuring manic symptomatology influences HPA axis alterations in depression. J. Psychiatr. Res. 45, 1208-1213. https://doi.org/10.1016/j.jpsychires. 2011.03.010.

Langan, C., McDonald, C., 2009. Neurobiological trait abnormalities in bipolar disorder. Mol. Psychiatry 14, 833-846. https://doi.org/10.1038/mp.2009.39.

Leverich, G.S., Nolen, W.A., Ruh, A.J., McElroy, S.L., Keck, P.E., Denicoff, K.D., et al, 2001. The Stanley foundation bipolar treatment outcome network. I. Longitudinal methodology. J. Affect. Disord. 67, 33-44. https://doi.org/10.1016/S0165-0327(01) 00430-X.

Linkowski, P., Kerkhofs, M., Van Onderbergen, A., Hubain, P., Copinschi, G., L'HermiteBalériaux, M., et al., 1994. The 24-hour profiles of cortisol, prolactin, and growth hormone secretion in mania. Arch. Gen. Psychiatry 51, 616-624.

Manenschijn, L., Koper, J.W., Lamberts, S.W., van Rossum, E.F., 2011. Evaluation of a method to measure long term cortisol levels. Steroids 76, 1032-1036. https://doi. org/10.1016/j.steroids.2011.04.005.

Maripuu, M., Wikgren, M., Karling, P., Adolfsson, R., Norrback, K.F., 2017. Hyper- and hypocortsicolis in bipolar disorder - a beneficial influence of lithium on the HPAaxis? J. Affect. Disord. 213, 161-167. https://doi.org/10.1016/j.jad.2017.02.026.

Noppe, G., de Rijke, Y.B., Dorst, K., van den Akker, E.L., van Rossum, E.F., 2015. LC-MS/ MS-based method for long-term steroid profiling in human scalp hair. Clin. Endocrinol. 83, 162-166. https://doi.org/10.1111/cen.12781.

Renes, J.W., Regeer, E.J., van der Voort, T.Y., Nolen, W.A., Kupka, R.W., 2014. Treatment of bipolar disorder in the Netherlands and concordance with treatment guidelines: study protocol of an observational, longitudinal study on naturalistic treatment of bipolar disorder in everyday clinical practice. BMC Psychiatry 14, 58. https://doi. org/10.1186/1471-244X-14-58.

Russell, E., Kirschbaum, C., Laudenslager, M.L., Stadler, T., de Rijke, Y., van Rossum, E.F., et al., 2015. Toward standardization of hair cortisol measurement: results of the first international interlaboratory round robin. Ther. Drug Monit. 37 (1), 71-75. https:// doi.org/10.1097/FTD.0000000000000148.

Sauvé, B., Koren, G., Walsh, G., Tokmakejian, S., Van Uum, S.H., 2007. Measurement of cortisol in human hair as a biomarker of systemic exposure. Clin. Invest. Med. 30, 183-191.

Schmider, J., Lammers, C.H., Gotthardt, U., Detting, M., Holsboer, F., Heuser, I.J., 1995 Combined dexamethasone/cortisotropin-releasing hormone test in acute and remitted manic patients, in acute depression and in normal controls. Biol. Psychiatry 38, 797-802. https://doi.org/10.1016/0006-3223(95)00064-X.

Sheehan, D.V., Lecrubier, Y., Sheehan, K.H., Amorim, P., Janavs, J., Weiller, E., Hergueta, T., Baker, R., Dunbar, G.C., 1998. The Mini-International Neuropsychiatric Interview (M.I.N.I.): the development and validation of a structured diagnostic psychiatric interview for DSM-IV and ICD-10. J. Clin. Psychiatry 59, 22-33.

Stadler, T., Kirschbaum, C., Alexander, N., Bornstein, S.R., Gao, W., Miller, R., et al., 2013. Cortisol in hair and the metabolic syndrome. J. Clin. Endocrinol. Metab. 98, 2573-2580. https://doi.org/10.1210/jc.2013-1056.

Staufenbiel, S.M., Penninx, B.W.J.H., de Rijke, Y.B., van den Akker, E.L.T., van Rossum, 
E.F.C., 2015. Determinants of hair cortisol and hair cortisone concentrations in adults. Psychoneuroendocrin 60, 182-194. https://doi.org/10.1016/j.psyneuen. 2015.06.011.

Streit, F., Memic, A., Hasandedic, L., Rietschel, L., Frank, J., Lang, M., et al., 2016. Perceived stress and hair cortisol: differences in bipolar disorder and schizophrenia. Psychoneuroendocrinology 69, 26-34. https://doi.org/10.1016/j.psyneuen.2016.03. 010.

Suppes, T., Leverich, G.S., Keck, P.E., Nolen, W.A., Denicoff, K.D., Altshuler, L.L., et al., 2001. The Stanley foundation bipolar treatment outcome network. II. Demographics and illness characteristics of the first 261 patients. J. Affect. Disord. 67, 45-59. https://doi.org/10.1016/S0165-0327(01)00432-3.

Van Uum, S.H., Sauvé, B., Fraser, L.A., Morley-Forster, P., Paul, T.L., Koren, G., 2008. Elevated content of cortisol in hair of patients with severe chronic pain: a novel biomarker for stress. Stress 11, 483-488. https://doi.org/10.1080/
10253890801887388.

Vieta, E., Martínez-De-Osaba, M.J., Colom, F., Martínez-Arán, A., Benabarre, A., Gastó, C. 1999. Enhanced corticotropin response to corticotropin-releasing hormone as a predictor of mania in euthymic bipolar patients. Psychol. Med. 29, 971-978.

Vreeker, A., Boks, M.P.M., Abramovic, L., Verkooijen, S., van Bergen, A.H., Hilligers,

M.H.J., et al., 2016. High educational performance is a distinctive feature of bipolar disorder: a study on cognition in bipolar disorder, schizophrenia patients, relatives and controls. Psychol. Med. 46, 807-818. https://doi.org/10.1017/ S0033291715002299.

Watson, S., Gallagher, P., Ritchie, J.C., Ferrier, N., Young, A.H., 2004.

Hypothalamic-pituitary-adrenal axis function in patients with bipolar disorder. Br. J. Psychiatry 184, 496-502.

Wester, V.L., van Rossum, E.F., 2015. Clinical applications of cortisol measurements in hair. Eur. J. Endocrinol. 173 (4), 1-10. https://doi.org/10.1530/EJE-15-0313. 